\title{
OPENTLM and SOCKET: Creating an Open EcoSystem for Virtual Prototyping of Complex SOCs
}

\author{
Laurent Maillet-Contoz \\ STMicroelectronics, Grenoble, France
}

\begin{abstract}
The objective of the OpenTLM project is to offer to embedded software developers a tool kit, available under open source license, and based on the SystemC/TLM standard. It enables them to develop and test the embedded software ahead of availability of hardware platforms (silicon, but also hardware emulators). It gives the opportunity to promote a broader use of the TLM methodology, already adopted by hardware teams, as well as a better concurrent development of hardware and software parts of the system. Indeed, if software is mature enough when silicon is available, the overall period for system integration is reduced, which accelerates the availability of the product and optimizes time-to-market.

The SoCKET project (SoC toolKit for critical Embedded sysTems) gathers industrial and academic partners to address the issue of design methodologies for critical embedded systems. The work targets the definition of a "seamless" design flow which integrates the equipment qualification/certification, from the system level to the Integrated Circuits (ICs) and the associated embedded software, compliant with the applicable norms (aeronautics: DO-178C, DO-254, ARP4754 space: ECSS Q60-02, Q80, E40).

This "seamless" flow requires some formalisms unification (elimination of semantic holes in HW/SW interfaces), the availability of models transformation operators (skeleton generation, requirements traceability), and models \& tools interoperability.
\end{abstract}

The main outcomes of the project will be:

* a design flow supporting critical embedded systems development

* a draft IDE implementing this flow and tested with partners's tools (adaptable with other tools and for other applications)

* some return of experience through 4 industrial case studies

* some Certification/Qualification kits for IPs and SoCs in each application domain

* some recommendations to certification and normalization bodies. 